\section{Agonizing obesity}

\section{By Tim Fulmer, Senior Writer}

Multiple cellular and hormonal signaling pathways often go awry in obesity, suggesting that simultaneously targeting various metabolic mechanisms might be a more efficient way to normalize body weight than focusing only on food intake.

A team led by researchers at Indiana University and the University of Cincinnati set out to design an antiobesity compound that would activate two distinct receptors known to play key roles in controlling body weight: glucagon-like peptide-1 receptor (GLP1R; GLP-1R) and glucagon receptor (GCGR). ${ }^{1}$ The group has now published mouse data for the coagonist peptides, which were exclusively licensed to Marcadia Biotech Inc., who in turn has licensed the molecules to Merck \& Co. Inc.

Activating GLP-1R is expected to reduce food intake by stimulating satiety centers in the CNS and slowing gastric emptying. ${ }^{2,3}$ Turning on GCGR should enhance energy expenditure by increasing the breakdown of hepatic glycogen and triggering lipolysis in adipose tissue.,

The group used the 29-amino-acid glucagon peptide as a scaffold and made successive amino acid substitutions to the peptide backbone. The result was two optimized peptide coagonists that both showed affinity for GLP-1R and GCGR in vitro.

The peptides were then pegylated to increase serum half-life, and an amino acid substitution with aminoisobutyric acid was made to provide resistance to dipeptidyl peptidase-4 (DPP-4), an endogenous enzyme that degrades GLP-1.

In mouse models of diet-induced obesity, a single subcutaneous injection of $325 \mathrm{nmol} / \mathrm{kg}$ of either coagonist compound decreased body weight, fat mass and average daily food intake at day 7 compared with injection of vehicle control.

The next step was testing the peptides in a chronic setting that more closely resembled how an obesity therapeutic might be given to patients. Over the course of a month, mice received an injection of one of the two peptides at a $70 \mathrm{nmol} / \mathrm{kg}$ dose once weekly.

By day 27, the 2 peptides significantly reduced body weight by $28.1 \%$ or $20.1 \%$ compared with vehicle control ( $p<0.0001$ for both). Mice in the treatment groups had decreases in fat mass of $62.9 \%$ or $52.2 \%$ compared with control mice ( $p<0.0001$ for both).

In addition, the reductions in weight corresponded to significant decreases in plasma levels of glucose $(p<0.0001)$, insulin $(p=0.0244)$ and cholesterol $(p=0.0441)$, as well as increased lipolysis in adipose tissue ( $p=0.0369)$ compared with what was seen using vehicle.

The $70 \mathrm{nmol} / \mathrm{kg}$ dose did not substantially alter food intake. However, mice given the molecules did have significantly greater overall energy expenditure than controls $(p=0.0187)$.
Overall, chronic administration of either coagonist peptide reduced weight, increased lipid metabolism in adipose tissue and improved glycemic control and insulin sensitivity.

The findings were published in Nature Chemical Biology.

\section{Making proper comparisons}

At least two GLP-1R agonists are in Phase III testing to treat obesity: Byetta exenatide synthetic exendin-4, from Amylin Pharmaceuticals Inc. and Eli Lilly and Co., and Victoza liraglutide, a long-acting analog of GLP-1 from Novo Nordisk A/S.

Byetta is approved in the U.S. and Europe to treat type 2 diabetes, whereas Victoza is approved for the indication in Europe. Over the course of those drugs' diabetes trials, it became apparent that GLP-1R agonists could induce sustained weight loss in some diabetics. ${ }^{6}$ All three companies declined to comment on the compounds described in the Nature Chemical Biology paper.

To better understand whether the coagonists described in the Nature Chemical Biology paper offer advantages over the GLP-1R agonists in the clinic, Michael Nauck wanted to see head-to-head comparisons of the compounds in obesity models. Nauck, who is a professor and senior physician at the Bad Lauterberg Diabetes Center, has published extensively on the use of GLP-1R agonists to treat type 2 diabetes.

"Since the paper's comparisons are to vehicle, it's difficult to know if the antiobesity effects observed are truly the result of coagonism at two distinct receptors or primarily the result of GLP-1R agonism," he said. "Answering that question in the same models should be straightforward using an active GLP-1R agonist comparator like exendin-4."

Genetic studies also could help illustrate the relative importance of GLP-1R and GCGR to the antiobesity effect of the coagonists, said David D'alessio, professor of endocrinology and staff physician at the University of Cincinnati, and a colleague of the paper's authors.

"The coagonists could be characterized in three separate groups of mice: glucagon receptor knockouts, Glp1r knockouts and wild-type controls," he said. "Comparisons of weight loss between those three groups should help reveal the relative contribution and importance of each receptor to the overall antiobesity effect."

The published results did provide clues that the improvements in obesity were not simply the result of GLP-1R agonism. In Glp1r knockout mice, one of the coagonists significantly decreased body weight, fat mass and food intake compared with vehicle $(p=0.0025, p=0.0025$ and $p=0.0017$, respectively).

Nevertheless, Brian Lavan, head of biology at Metabolex Inc., wanted to see the coagonist peptides characterized in animal models that more closely resemble chronic obesity in humans.

"The researchers clearly see effects in the diet-induced obesity model. However, chronic obesity in humans is often accompanied by long-standing impaired glucose metabolism, with aberrantly increased levels of plasma insulin and glucagon," he said. "Based on what's in the paper, it's not clear that coagonizing the glucagon receptor would have an antiobesity effect in the presence of significant hyperglucagonemia, where potentially impaired signaling through the glucagon receptor could reduce the effi- 


\section{TARGETS \& MECHANISMS}

cacy of the coagonist."

Lavan therefore suggested looking at the coagonist peptides in diabetic mouse models that show chronic glucose dysregulation, with long-standing hyperinsulinemia and hyperglucagonemia.

Metabolex's MBX-2982, a small molecule G protein-coupled receptor 119 (GPR119) agonist that triggers release of GLP-1 from intestinal L cells and insulin from pancreatic islet cells, ${ }^{7}$ is in Phase I testing to treat type 2 diabetes.

\section{Merck double dips}

Marcadia in-licensed the IP covering the coagonist peptides from Indiana University. Last year, the biotech began a collaboration with Merck to develop and commercialize the compounds to treat obesity and diabetes. The pharma received exclusive worldwide rights to peptide co-agonists designed by Marcadia. In return, the biotech receives licensing fees and research funding.

Merck is also working on a second type of GLP-1R/GCGR coagonist peptide. In a paper published last month in Diabetes, ${ }^{8}$ the company reported on coagonists based on the endogenous gut peptide oxyntomodulin, which binds both receptors in its wild-type form and reduces food intake and increases energy expenditure in rodents. ${ }^{9}$

In mouse models of diet-induced obesity, an oxyntomodulin-based coagonist caused greater reductions of body weight, fat mass and average food intake than both vehicle and a GLP-1R agonist.

Moreover, in separate groups of Glpr1 and Gcgr knockout mice, the antiobesity effects of the coagonist were partially reduced compared with those in wild-type mice. That result helped confirm that agonism of both GLP-1R and GCGR was required for the full effect.

Ranabir SinhaRoy, a principle investigator on the Diabetes paper and team leader of the biology program in Merck's diabetes and obesity franchise, told SciBX he is now interested in evaluating the oxyntomodulinbased coagonist in human trials.

Fritz French, CEO of Marcadia, said his company is now optimizing the chemical structures of the glucagon-based coagonists to improve safety and efficacy.

Fulmer, T. SciBX 2(31); doi:10.1038/scibx.2009.1200

Published online Aug. 13, 2009

\section{REFERENCES}

1. Day, J. et al. Nat. Chem. Biol.; published online July 13, 2009; doi: $10.1038 /$ nchembio. 209

Contact: Richard DiMarchi, Indiana University, Bloomington, Ind. e-mail: rdimarch@indiana.edu

2. Holst, J. J. Physiol. Rev. 87, 1409-1439 (2007)

3. Gallwitz, B. Rev. Diabetic Stud. 2, 61-69 (2005)

4. Woods, S. et al. Phil. Trans. R. Soc. B 361, 1219-1235 (2006)

5. Silva, J. Physiol. Rev. 86, 435-464 (2006)

6. Amori, R. et al. JAMA 298, 194-206 (2007)

7. Overton, H. et al. Br. J. Pharmacol. 153, S76-S81 (2008)

8. Pocai, A. et al. Diabetes; published online July 14, 2009; doi:10.2337/db09-0278

9. Baggio, L. et al. Gastroenterol. 127, 546-558 (2004)

\section{COMPANIES AND INSTITUTIONS MENTIONED}

Amylin Pharmaceuticals Inc. (NASDAQ:AMLN), San Diego, Calif. Bad Lauterberg Diabetes Center, Bad Lauterberg im Harz, Germany Eli Lilly and Co. (NYSE:LLY), Indianapolis, Ind.

Indiana University, Bloomington, Ind.

Marcadia Biotech Inc., Carmel, Ind.

Merck \& Co. Inc. (NYSE:MRK), Whitehouse Station, N.J.

Metabolex Inc., Hayward, Calif.

Novo Nordisk A/S (CSE:NVO; NYSE:NVO), Bagsvaerd, Denmark University of Cincinnati, Cincinnati, Ohio 